Supporting information for the article

\title{
New device and method for flux-proportional sampling of mobile solutes in soil and groundwater
}

\author{
Hubert de Jonge* ${ }^{*}, b$ and Gadi Rothenberg ${ }^{c}$ \\ ${ }^{a}$ Danish Institute of Agricultural Sciences, Research Centre Foulum, P.O. Box 50, DK-8830 Tjele, Denmark. Fax \\ +458999 1719, e-mail Hubert.deJonge@agrsci.dk \\ ${ }^{b}$ Sorbisense, Agro Business Park, Niels Pedersens Allé 2, DK-8830 Tjele, Denmark. \\ ${ }^{c}$ van 't Hoff Institute for Molecular Sciences, University of Amsterdam, Nieuwe Achtergracht 166, $1018 \mathrm{WV}$ \\ Amsterdam, The Netherlands.
}

Chemicals. 1-dodecylamine, 98\%, Aldrich D22,220-8; Ethanol, EtOH absolute, 99.9\%, Merck; Methanol, MeOH, HPLC grade 99.8\%, J.T. Baker; Ammonium heptamolybdate, Aldrich 22,123-6; Tetraethoxysilane, TEOS, 98\%, Aldrich 13,190-3; ${ }^{14} \mathrm{C}$ Phenanthrene (ARC-867, Biotrend, Köln, Germany); ${ }^{12} \mathrm{C}$ Phenanthrene 98\%, Aldrich P1,140-9; Ethyl-acetate 99.99\%, HPLC grade, Bie\&Berntsen 40005, Denmark; Carbotrap C, Supelco 20309; Amberlite XAD-7, BDH 152444R.

Preparation of Mo-substituted hexagonal mesoporous silica (Mo-HMS, adsorbent B). 5.08 gram of 1-dodecylamine was dissolved in $46.00 \mathrm{~mL}$ absolute EtOH and $0.497 \mathrm{~g}$ ammonium heptamolybdate was dissolved in $53.00 \mathrm{~mL}$ demineralized water. The EtOH solution was placed in a $500 \mathrm{~mL}$ round-bottomed flask with an egg-shaped stirrer bar. Then, $20.13 \mathrm{~mL}$ of tetraethoxysilane (TEOS) and the heptamolybdate solution were added and mixture was gently swirled for $10 \mathrm{~min}$. A milky white suspension formed, which was stirred overnight at $500 \mathrm{rpm}$. The resulting white cake was then washed in EtOH $(2 \times 25 \mathrm{~mL})$. The powder was then extracted for $24 \mathrm{~h}$ in EtOH using a soxhlet apparatus, and dried at $105^{\circ} \mathrm{C}$ for $4 \mathrm{~h}$. A portion of the material was calcined at $700{ }^{\circ} \mathrm{C}$ for $16 \mathrm{~h}$.

Setup for long-term soil column experiments. Figure S1 shows a schematic of the soil column used in the long-term leaching studies of phenanthrene and glyphosate. Acid-rinsed, 10 $\mathrm{L}$ brown glass bottles were used to store input and effluent solutions. The fluid pumps were of type RHSY (FMI, Syosset, NY). The capillaries used were either stainless steel or PTFE.A twoway valve was positioned between the pump and the irrigation head to allow sampling of the input solution. The irrigation head was constructed of stainless steel and was equipped with 29 
needles (Microlance 3, Droghede, Ireland). A fine nylon mesh was placed on the soil surface to facilitate a uniform distribution of the input solution. Unsaturated conditions at the bottom of the column were maintained by a hanging a $35 \mathrm{~cm}$ water column between the bottom of the soil column and the outlet.

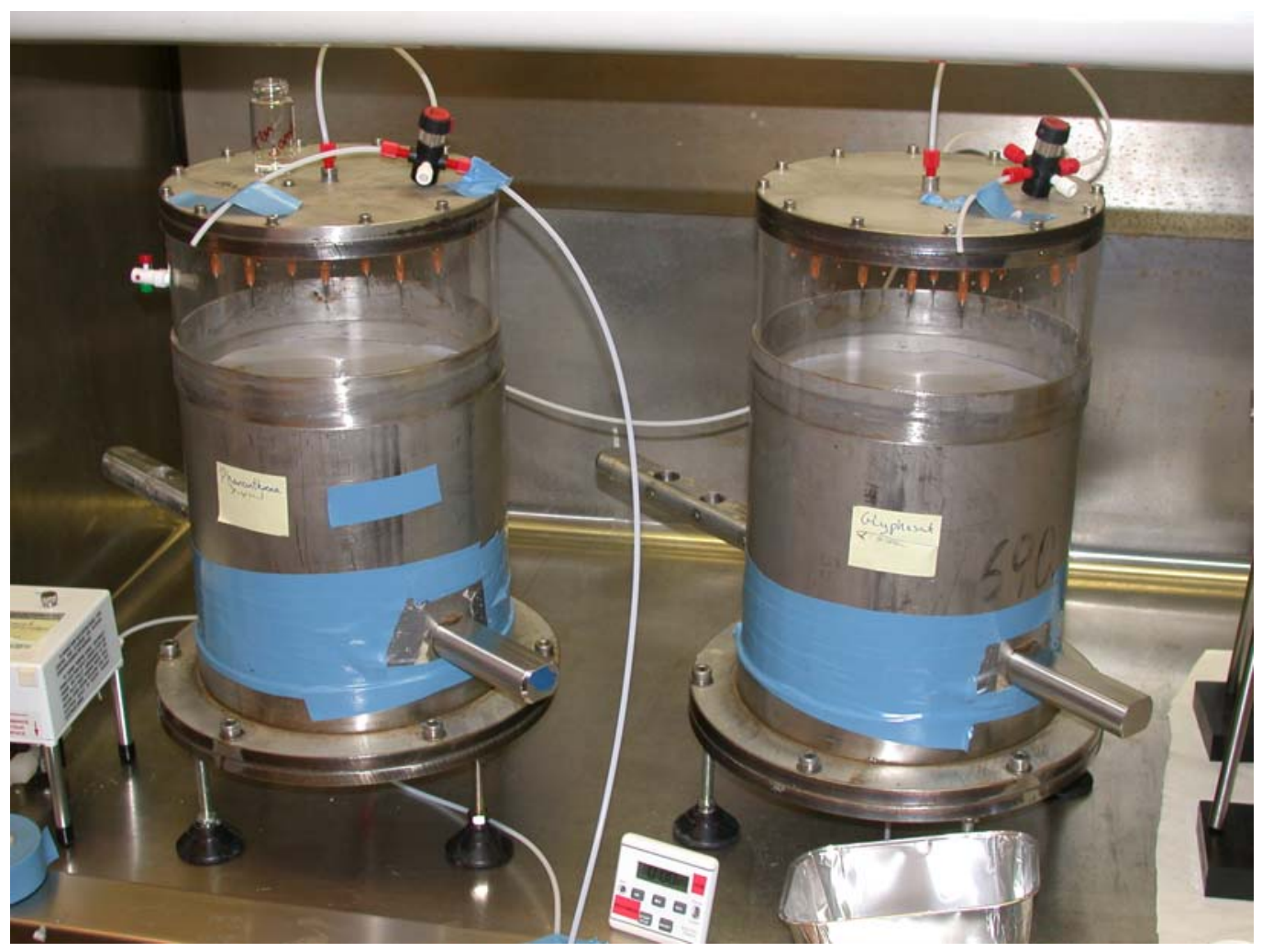

Figure S1. Photo of the soil columns and sampler prototypes used for long-term transport and leaching studies of PHEN (left column) and GLY (right column). Each sampler is made from 316L grade stainless steel and has six vertical holes wherein precisely machined stainless steel cylindrical cartridges (inner diameter $14.2 \mathrm{~mm}$, height $17.1 \mathrm{~mm}$ ) are fitted. These cartridges are packed with adsorbents. The total volume of the packed bed of adsorbent in each cartridge is $2.71 \mathrm{~cm}^{3}$. The cartridges are closed on both sides with stainless steel frits (grade 40 stainless steel, diameter $15.9 \mathrm{~mm}$, thickness $2.5 \mathrm{~mm}$ ) purchased from Porvair (Wrexham, UK).

Procedure for saturated hydraulic conductivity measurements. Each cartridge was rinsed with acetone and demineralized water, filled with adsorbent, closed with steel frits and connected to a Marriot flask. A constant head pressure of $50 \mathrm{~mm}$ was applied, and the outflow at steady state was recorded. This procedure was repeated at head pressures of $100 \mathrm{~mm}$ and 150 
mm. The hydraulic conductivity, $K$, was calculated from $K=\frac{V \cdot d z}{A \cdot t \cdot d h}$, wherein $V / t$ is the flow rate, $A$ is the cross section of the cartridge, $d z$ is the total height of the cartridge including steel frits, and $d h$ is the head pressure.

Tracer transport and the effect of diffusion The displacement of solute mass in variously saturated porous media occurs by the combination of convection, $J_{m}$, diffusion, $J_{D}$, and hydrodynamic dispersion, $J_{h}$ (Van Genuchten en Wierenga, 1986). Steady state liquid flow in a homogenous porous medium is the transport equation is given by the transport equation:

$$
\frac{\partial C}{\partial t}=\frac{D \partial^{2} C}{R \partial x^{2}}-\frac{q \partial C}{R \partial x}
$$

where $C$ is the solute concentration $\left(\mathrm{g} / \mathrm{cm}^{3}\right), t$ is time, $D$ is the effective dispersion coefficient, $R$ is the sorption retardation factor, $x$ is the space coordinate, $q$ is the volumetric flux density $(\mathrm{cm} / \mathrm{s})$, Solving analytically ${ }^{1}$ for the boundary conditions $\left\{C(x, 0)=0 ; C(0, t)=C_{0} H(t)\right.$; $C(\infty, t)=0\}$, where $C_{0}$ is the concentration at the interface $(x=0)$, and $H$ is the heavyside step function, and assuming a semi-infinite length of the porous medium, we obtain eq 2. Here, erfc is the complementary error function, and $x$ is the distance increasing in the flow direction.

$$
\frac{C(x, t)}{C_{0}}=\frac{1}{2} \operatorname{erfc}\left(\frac{R x-q t}{2 \sqrt{D R t}}\right)+\frac{1}{2} \exp \left(\frac{q x}{D}\right) \operatorname{erfc}\left(\frac{R x-q t}{2 \sqrt{D R t}}\right)
$$

The tracer can be placed in the centre section of the porous cartridge (Figure 1 in the main document, right, illuvation measurement). One can measure the increase of the tracer in both the upstream and downstream compartment. These compartments are filled with an adsorbent that can sequester the tracer. This configuration can compensate for tracer displacement due to diffusion. For upstream diffusion of a solute, eq 2 still applies, except that $q$ is negative, i.e. $q_{\text {upstream }}=-q_{\text {downstream }}$. If all the tracer that is displaced into the adsorbent section is sequestered in the adsorbent section, the sampler can be regarded as a semi-infinite porous medium, and the net result of diffusion to the displacement of the centre of mass is zero. Hence, a very useful property may be derived by numerically integrating equation 2 for the case of downstream and upstream displacement. It follows that,

(1). See e.g. van Genuchten, M. Th., Wierenga, P. J., In Methods of soil analysis. Part 1. Physical and Mineralogical Methods. Klute, A. (Ed.), ASA: Madison, Wisconsin, (1986). 


$$
q=\frac{R}{\left(C_{t \max } T\right)}\left(\sum_{x=0}^{l} C_{x} \Delta x-\sum_{x=-l}^{0} C_{x} \Delta x\right) \equiv \frac{R}{\left(C_{t \text { max }} T\right)}\left(m_{t 1}-m_{t 2}\right)
$$

Where $l$ is the distance from the source of the tracer to the end of the adsorbent section, $m_{t l}$ and $m_{t 2}$ representing the tracer mass in solution displaced in the downstream and upstream direction, respectively, with the unit $\mathrm{g} / \mathrm{cm}^{2}$, and $\mathrm{T}$ is sampling time Substituting $q=V / A T$ we obtain eq 4, where $M_{t 1}$ and $M_{t 2}$ is the total tracer mass (in grams) displaced in downstream and upstream direction, respectively, and $A$ is the cross-section area of the cartridge.

$$
V=\frac{R A}{C_{t \max }}\left(m_{t 1}-m_{t 2}\right)=\frac{\left(M_{t 1}-M_{t 2}\right)}{C_{t \max }}
$$

Thus, $V$ may be derived from the mass of a tracer that is displaced upstream and downstream during the installation period, irrespective of the porewater velocity $q$. It follows from equation 4 that $V=0$ when $M_{t 1}=M_{t 2}$ (fully diffusion controlled). When $M_{t 2} \rightarrow 0$, the solute displacement is fully controlled by convective transport. 